\title{
Idiopathic pleuroparenchymal fibroelastosis: an unrecognized or misdiagnosed entity?
}

\author{
Christian D Becker ${ }^{1}$, Joan Gil ${ }^{2}$ and Maria L Padilla ${ }^{1}$ \\ ${ }^{1}$ Department of Medicine, Division of Pulmonary, Critical Care and Sleep Medicine, The Mount Sinai School \\ of Medicine, New York, NY, USA and ${ }^{2}$ Department of Pathology, The Mount Sinai School of Medicine, \\ New York, NY, USA
}

\begin{abstract}
Idiopathic pleuroparenchymal fibroelastosis is a rare recently described entity likely to be under- and misdiagnosed, as awareness of this entity is not yet widespread. We report two cases that show the need to include this disease in the differential diagnosis of patients with predominantly pleural and subpleural fibrotic processes. The condition is a fibrotic thickening of the pleura and subpleural parenchyma due to elastic fiber proliferation predominantly in the upper lobes. Performing elastic fiber stains routinely in patients with fibrosis of this distribution may, therefore, aid in establishing the diagnosis and differentiating it from usual interstitial pneumonia/idiopathic pulmonary fibrosis. These patients may be prone to the development of secondary spontaneous pneumothoraces and persistent postoperative bronchopleural fistulae. Continued study of newly diagnosed cases may uncover shared characteristics or features helpful in generating an etiologic hypothesis. Only with better understanding of this disease can we hope in the future to be able to offer treatments other than supportive care and ultimately lung transplantation, which are the only therapeutic options available today.
\end{abstract}

Modern Pathology (2008) 21, 784-787; doi:10.1038/modpathol.2008.56; published online 11 April 2008

Keywords: interstitial lung disease; pulmonary fibrosis; pleural fibrosis; fibroelastosis (not MeSH)

\section{Case 1}

A 51-year-old woman presented with progressive dyspnea on exertion and non-productive cough worsening despite aggressive treatment. Her exercise limitation was less than 1 block or 1/2 flight of stairs. Her past medical history included emphysema, hypertension, hyperlipidemia and gastroesophageal reflux disease. Her medications included supplemental $\mathrm{O}_{2}$ prn, tiotropium bromide, inhaled budesonide and formoterol fumarate, montelukast, theophylline prn, prednisone, omeprazole, amlodipine, diltiazem and atorvastatin. She reported no ergot alkaloid use, illicit drug use or toxic exposures. She was an ex-smoker and used alcohol socially. Pulmonary function testing showed a severe obstructive ventilatory impairment with a mild gas transfer defect. Her chest X-ray is shown in Figure 1, her chest CT in Figure 2. She underwent bronchoscopy,

Correspondence: Dr CD Becker, MD, PhD, Department of Medicine, Division of Pulmonary, Critical Care and Sleep Medicine, The Mount Sinai School of Medicine, One Gustave L. Levy Place, Box 1232, New York, NY 10029, USA.

E-mails: Christian.becker@mssm.edu or

Christian.d.becker@gmail.com

Received 04 November 2007; revised 06 March 2008; accepted 10 March 2008; published online 11 April 2008 video-assisted thoracoscopy with extensive pleurolysis and wedge resections of the right upper lobe, right middle lobe and right lower lobe. The procedure was complicated by a persistent right-sided pneumothorax with bronchopleural fistula, which resolved post-operatively. The slides obtained during the procedure were diagnosed as idiopathic pleuroparenchymal fibroelastosis (Figure 3).

\section{Case 2}

A 59-year-old woman presented to her pulmonologist with a complaint of worsening dyspnea on exertion and non-productive cough. Her past medical history was significant for chronic hyponatremia, mild intermittent asthma, hiatal hernia and depression. Her medications included prednisone, inhaled fluticasone/salmeterol and tiotropium bromide, mesomeprazole and duloxetine hydrochloride. She reported possible asbestos exposure in childhood and had smoked in the past. She reported no alcohol or illicit drug use. She was employed as a librarian. A follicular center cell lymphoma had been diagnosed in 1996 and she had undergone extensive chemotherapy (fludarabine (refractory), CHOP and rituximab in 2002, autologous stem cell transplant in 2002 and ibritumomab tiuxetan in 


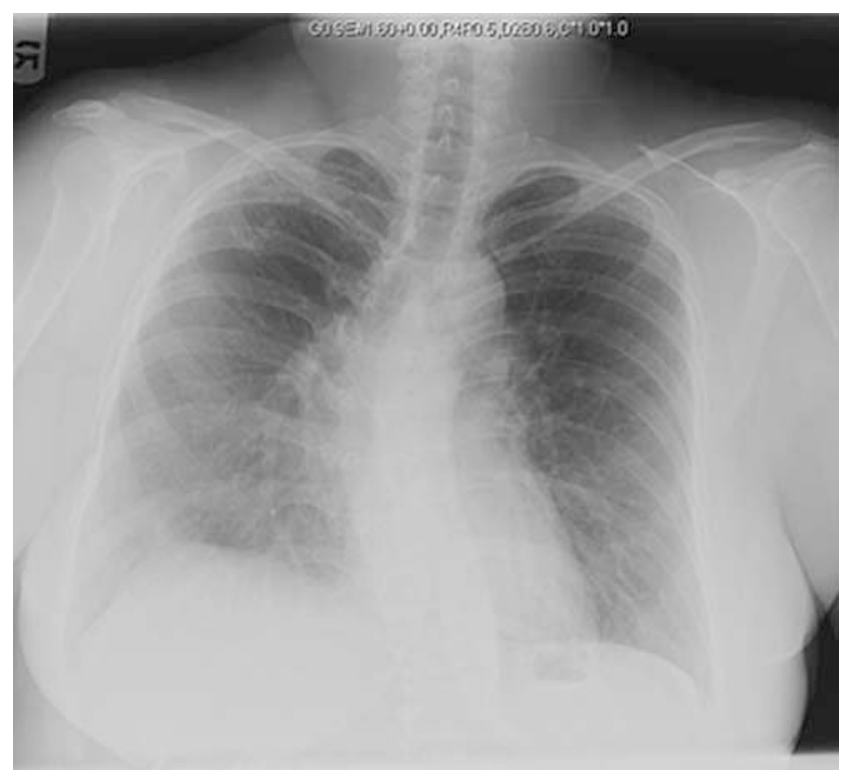

Figure 1 Case 1 chest X-ray: biapical fibrotic changes with some nodular components. Tenting of the right hemidiaphragm and upward retraction of the right hilum.

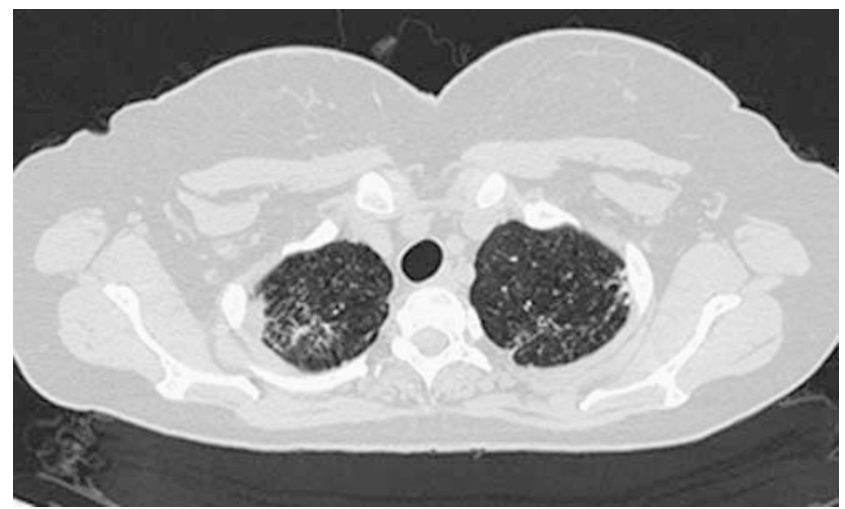

Figure 2 Case 1 chest CT: biapical fibrotic changes, pleural and parenchymal densities in the right upper lobe as well as mild emphysematic changes.

2005). Pulmonary function testing showed a moderate restrictive ventilatory impairment and a mild gas transfer defect. Infiltrative changes on her chest X-ray triggered a PET/CT scan, which showed a secondary spontaneous right-sided pneumothorax and apical pleural fibrotic changes (Figure 4). She underwent video-assisted thoracoscopy with wedge biopsies of the right upper, middle and lower lobes and pleural abrasion at another hospital. The procedure was complicated by a large bronchopleural fistula. The pathological specimen was originally interpreted as consistent with usual interstitial pneumonia/idiopathic pulmonary fibrosis, without any evidence of asbestos fibers. Elastic fiber stains were not performed. Her bronchopleural fistula persisted and she was transferred to our hospital for revision, decortication, pleurodesis and pleural tenting (her chest X-ray on arrival at this hospital is shown in Figure 5). The pleural surface

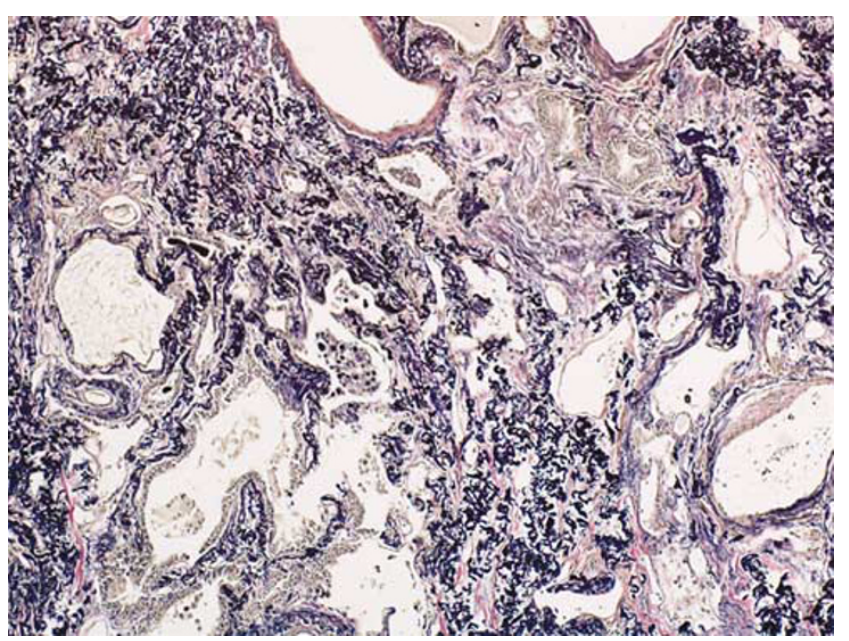

Figure 3 Case 1 histopathology: elastic fiber stain (Elastic Van Gieson) demonstrating abundance of short, curled and randomly oriented elastic fibers in the thickened interstitium.

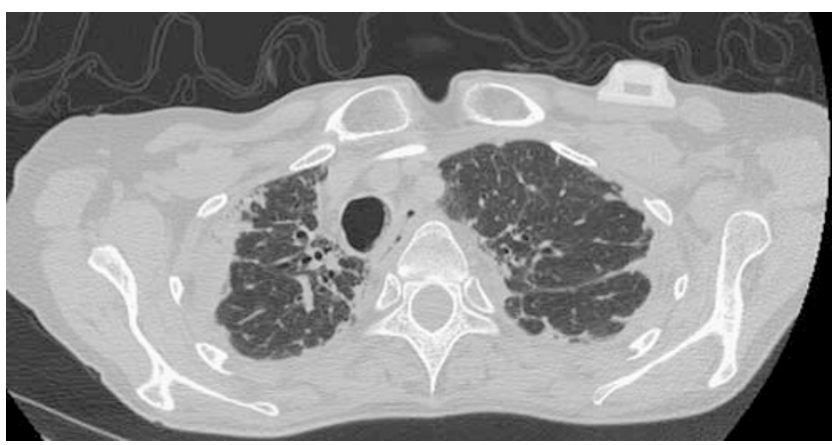

Figure 4 Case 2 chest CT: biapical pleural fibrotic changes, more prominent on the right. Subcutaneous portacath reservoir left anterior chest wall.

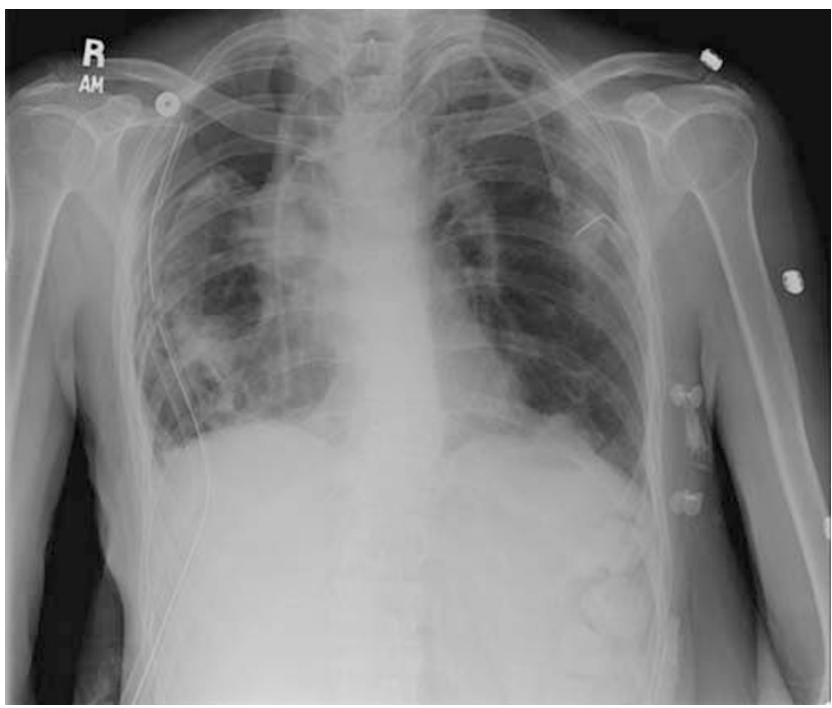

Figure 5 Case 2 chest X-ray: left-sided portacath, right-sided chest tube, large right sided pneumothorax, thickening of the right visceral pleura, patchy atelectasis, surgical staples in right lung. 


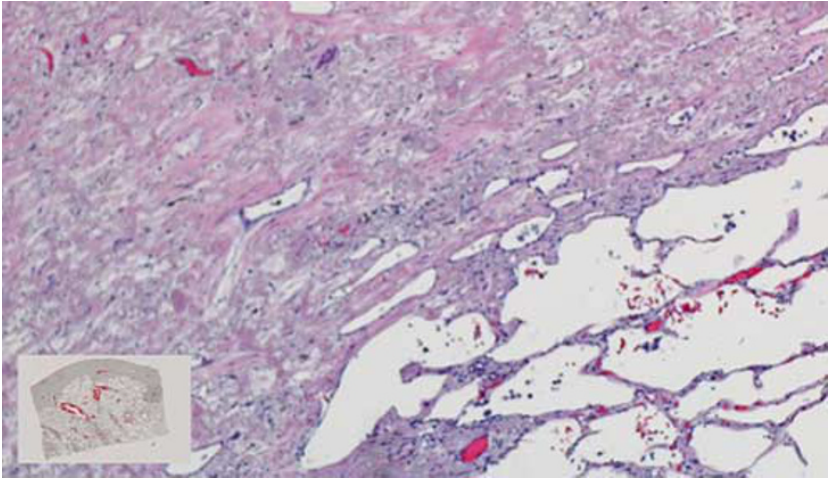

Figure 6 Case 2 pathology: hematoxylin-eosin stain showing abundance of elastic fibers in addition to collagen fibers and abrupt transition from fibroelastosis to unaffected normal lung parenchyma. Insert: slide 'thumbnail' view demonstrating an extraordinarily thick pleural cap with sparing of the adjacent lung parenchyma.

was found to be very thick and fibrotic, forming ubiquitous adhesions, encasing the right upper regions of the lung and hindering expansion of the remaining lung tissue. The procedure was again complicated by a large bronchopleural fistula, which persisted despite multiple operative revisions. She developed septic complications and required mechanical ventilation and tracheostomy. Her condition deteriorated and she passed away. The outside slides were reviewed and the diagnosis was amended to idiopathic pleuroparenchymal fibroelastosis (Figure 6).

\section{Discussion}

Idiopathic pleuroparenchymal fibroelastosis was first described in 2004 by the Interstitial Lung Disease Program of the National Jewish Medical and Research Center, Denver, CO, USA. ${ }^{1}$ The group reported five patients with a unique pathologic picture of pleural fibrosis and subpleural parenchymal fibrosis, mostly in the upper lobes, which did not fit any of the currently defined categories of idiopathic interstitial pneumonias in that the fibrosing injuries consisted of elastic fibers. This differs from the usual forms of fibrosis, which almost invariably show collagenous predominance. We are not aware of additional cases reported in the literature. The unique differentiating pathologic features of idiopathic pleuroparenchymal fibroelastosis are intense, predominantly elastic fibrosis of the visceral pleura, particularly of the upper lobes, which consists of accumulations of short, curled and randomly oriented elastic fibers (as seen in Figure 3). The entire pleura appears as a homogeneously thick elastic band. The elastosis is seen extending into the alveolar walls that insert in the pleura and beyond, although in our cases it never occupied the whole extent of the sections obtained. The transition from thickened, elastic alveolar walls to normal parenchyma was sharp. Despite the severity of the presentation, our cases did not display the fibrotic remodeling characteristic of usual interstitial pneumonia/idiopathic pulmonary fibrosis (honeycombing in particular). We noticed small, to an extent, questionable fibroblastic foci in the superficial biopsies of the parenchyma received. Overall, the histologic picture was more temporally homogeneous as opposed to the temporally heterogeneous picture seen in usual interstitial pneumonia/ idiopathic pulmonary fibrosis. There were some mild interstitial lymphocytic infiltrates. Histologically, the features, in particular the elastic band-like thickening of the pleura, were easily recognizable, but in case of doubt, an elastin stain (EVG in our case) will resolve the differential diagnosis. Radiographically there was intense pleural thickening and fibrosis with an upper lobe predominance. Hilar retractions and upper lobe volume loss may also be seen.

The differential diagnosis for the relatively rare combination of both pleural and parenchymal fibrosis includes asbestosis, connective tissue diseases, advanced fibrosing sarcoidosis and radiation or drug induced lung disease. On biopsy the feature unique to idiopathic pleuroparenchymal fibroelastosis is the predominance of elastic fibers. Idiopathic pleuroparenchymal fibroelastosis may be misdiagnosed as usual interstitial pneumonia/ idiopathic pulmonary fibrosis.

In our second case, the surgical findings during thoracoscopy as well as the radiographic findings of a mainly pleural and immediately subpleural parenchymal process (Figure 4) raised suspicion for the diagnosis of idiopathic pleuroparenchymal fibroelastosis, but elastic fiber stains had not been performed by the original pathologists. Performing elastic fiber stains routinely in cases of predominantly pleural and subpleural fibrosis may aid greatly in establishing the diagnosis.

It is difficult to understand why pleuroparenchymal fibroelastosis seems to predispose patients to secondary spontaneous or iatrogenic pneumothoraces. Maybe the elastic pleura offers low resistance to tear. In addition, surgically injured affected tissue with bronchopleural fistulae may exhibit a limited healing capability. Both of our patients did develop these complications, case 1 as a procedural complication and case 2 as a secondary spontaneous pneumothorax. They posed a therapeutic challenge in both cases. Thoracic surgeons should be aware of the unique pathologic features of this entity and adapt their procedural approach accordingly. It is likely that this poorly understood and rare, but severe condition is currently underdiagnosed. The frequency of diagnosis may increase in the near future.

The etiology and pathophysiology of this entity are unknown. The pathologic and radiographic features of the disease may result from a specific defect or represent a reactive pattern of the lung to various injuries, which may be influenced by genetic factors.

A murine model of TGF- $\alpha$ upregulation ${ }^{2,3}$ causes pleural and parenchymal fibrosis with markedly increased elastin expression as shown by microarray studies $^{4}$ and, thus, may help to understand the 
underlying disease mechanisms. A hereditary factor in idiopathic pleuroparenchymal fibroelastosis is conceivable, as the original report included one affected sib pair. Whether empiric treatment with TGF- $\alpha$ inhibitors is an option is uncertain at this time. The high symptom burden and the lack of other available therapies may make this a treatment option of last resort. Currently, there is no effective treatment and ultimately the only option for these patients is lung transplantation.

\section{Conflict of interest}

The authors state no conflict of interest.

\section{References}

1 Frankel SK, Cool CD, Lynch DA, et al. Pleuroparenchymal fibroelastosis: description of a novel clinicopathological entity. Chest 2004;126:2007-2013.

2 Hardie WD, Piljan-Gentle A, Dunlavy MR, et al. Dose-dependent lung remodeling in transgenic mice expressing transforming growth factor $\alpha$. Am J Physiol Lung Cell Mol Physiol 2001;281:L1088-L1094.

3 Hardie WD, Le Gras TD, Jiang K, et al. Conditional expression of transforming growth factor $\alpha$ in adult mouse lung causes pulmonary fibrosis. Am J Physiol Lung Cell Mol Physiol 2004;286:L741-L749.

4 Hardie WD, Personal communication Children's Hospital Medical Center: Cininnati, Ohio. 